\title{
QUALIDADE DO AR - PARÂMETROS DE CONTROLE E EFEITOS NA SAÚDE HUMANA: UMA BREVE REVISÃO
}

\author{
A. H. S. Castro ${ }^{1}$, R. S. Araújo ${ }^{2}$ G. M. M. SILVA ${ }^{3}$
}

Programa de Pós Graduação em Tecnologia de Gestão Ambiental - IFCE E-mail: hermeson.ifce@gmail.com ${ }^{1}$, gloriamarinho@ifce.edu.br ${ }^{2}$, rinaldo@ifce.edu.br ${ }^{3}$

Artigo submetido em janeiro/2013 e aceito em outubro/2013

\section{RESUMO}

Estudos relacionados à poluição atmosférica e a qualidade do ar crescem a cada ano. Em geral as pesquisas desenvolvidas envolvem o conhecimento sobre os componentes nocivos a saúde presentes no meio ambiente. De acordo com a legislação brasileira os poluentes de maior importância monitorados são representados pelo monóxido de carbono (CO), os óxidos de nitrogênio $\left(\mathrm{NO}_{\mathrm{x}}\right)$, o dióxido de enxofre $\left(\mathrm{SO}_{2}\right)$, o ozônio $\left(\mathrm{O}_{3}\right)$, a fumaça e os materiais particulados: partículas inaláveis $\left(\mathrm{PM}_{10}\right.$ e $\left.\mathrm{PM}_{2,5}\right)$ e partículas totais em suspensão (PTS). Outras substâncias como os compostos orgânicos BTEX (benzeno, tolueno, etilbenzeno e xilenos) também foram investigados devido à elevada abundancia em atmosferas urbanas. Em particular, este artigo apresenta uma revisão sobre os principais poluentes atmosféricos, fontes de emissão, ocorrência, legislação e efeitos sobre a saúde humana.

PALAVRAS-CHAVE: Qualidade do Ar, Poluentes Atmosféricos, Legislação e Saúde.

\section{AIR QUALITY - PARAMETERS OF CONTROL AND EFFECTS ON HUMAN HEALTH: A BRIEF REVIEW}

\section{ABSTRACT}

Studies related to air pollution and air quality increase every year. In general the researches are related to the knowledge about human health harmful components present in the environment. In according to Brazilian legislation the most important pollutants monitored are represented by carbon monoxide (CO), nitrogen oxides $\left(\mathrm{NO}_{\mathrm{x}}\right)$, sulfur dioxide $\left(\mathrm{SO}_{2}\right)$, ozone $\left(\mathrm{O}_{3}\right)$, smoke and particulate matter: inhalable particles $\left(\mathrm{PM}_{10}\right.$ and $\left.\mathrm{PM}_{2,5}\right)$ and total suspended particulates (TSP). Other substances such as organic compounds BTEX (benzene, toluene, ethylbenzene and xylenes) were also investigated due to their high abundance in urban atmospheres. In particular, this paper presents a review of the main air pollutants, emission sources, occurrence, legislation and effects on human health.

KEYWORDS: Air Quality, Atmospheric Pollutants, Legislation and Health. 


\section{QUALIDADE DO AR - PARÂMETROS DE CONTROLE E EFEITOS NA SAÚDE HUMANA: UMA BREVE REVISÃO}

\section{INTRODUÇÃO}

De acordo com a ideia de desenvolvimento sustentável, a poluição ambiental destaca-se como um dos principais aspectos a ser discutido por pesquisadores, entidades governamentais e pela sociedade em geral (HORGNIES et. al., 2012). Nas últimas décadas vem sendo registrado um significativo aumento nas concentrações de substâncias perigosas no ambiente atmosférico, o que é motivo de preocupação global. Tal fato é decorrente de atividades desenvolvidas pela espécie humana. Poluentes atmosféricos causam efeitos verdadeiramente adversos, principalmente em cidades com elevado grau de urbanização e atividade industrial.

Os veículos automotores e as atividades industriais estão ligados diretamente a problemas ambientais e de saúde pública, uma vez que as emissões veiculares, juntamente com as emissões de fontes estacionárias, são as principais responsáveis pela presença dos mais variados compostos na atmosfera. Dentre os poluentes que apresentam maior importância na química da baixa troposfera, podemos citar: o monóxido de carbono (CO), os óxidos de nitrogênio $\left(\mathrm{NO}_{\mathrm{x}}\right)$, o dióxido de enxofre $\left(\mathrm{SO}_{2}\right)$, o ozônio $\left(\mathrm{O}_{3}\right)$, a fumaça e os materiais particulados, representados pelas partículas inaláveis $\left(\mathrm{PM}_{10}\right.$ e $\left.\mathrm{PM}_{2,5}\right)$ e pelas partículas em suspensão.

Adicionalmente, destacam-se as substâncias decorrentes da evaporação/combustão dos combustíveis fósseis, representadas principalmente pelos compostos orgânicos voláteis (COVs) e os hidrocarbonetos poliaromáticos (HPAs). Poluentes gasosos são reconhecidos por causar danos à saúde, essencialmente em indivíduos com doenças pré-existentes. Em particular, compostos orgânicos voláteis (VOCs) são altamente reativos, possuem elevado caráter tóxico e podem participar de inúmeras reações na atmosfera formando poluentes secundários (CASELLI et. al., 2010).

Particularmente, BTEX (benzeno tolueno, etilbenzeno e xilenos) quando presentes em áreas urbanas constituem um dos principais problemas para a qualidade de vida humana, não só pela sua contribuição significativa na formação de oxidantes, como ozônio e nitrato peroxiacetilo (PAN), mas também por seus efeitos adversos sobre a saúde humana (ZHANG et. al., 2012).

Nos últimos anos, os estudos envolvendo campanhas de controle da qualidade do ar nas zonas urbanas vêm crescendo, e muitos têm foco no monitoramento de benzeno, tolueno, etilbenzeno e os isômeros do xileno ou nos compostos orgânicos voláteis (MURENA, 2007).

Neste contexto, é objetivo deste trabalho produzir uma breve revisão referente à qualidade do ar e os principais poluentes atmosféricos. Serão abordados aspectos referentes à química destes poluentes, ao modo como são emitidos no ar, a legislação inerente e aos efeitos sobre a saúde humana.

\section{POLUENTES CLÁSSICOS: $\mathrm{NO}_{\mathrm{x}}, \mathrm{SO}_{2}, \mathrm{MP}$}

\section{1. Óxidos de Nitrogênio $\left(\mathrm{NO}_{\mathrm{x}}\right)$}


Os óxidos de nitrogênio $\left(\mathrm{NO}_{\mathrm{x}}\right)$ são compostos poluentes gasosos comuns e tóxicos que afetam a saúde de milhões de seres humanos, especialmente em áreas urbanas. Os " $\mathrm{NO}_{\mathrm{x}}$ " representam duas espécies moleculares: o dióxido de nitrogênio $\left(\mathrm{NO}_{2}\right)$ e o monóxido de nitrogênio (NO) (HORGNIES et. al., 2012). Os óxidos de nitrogênio são compostos provenientes de uma série de atividades antrópicas e naturais como: a queima de combustíveis fósseis, a combustão de biomassa, a utilização de fertilizantes, os processos industriais e até mesmo fenômenos naturais (HORGNIES et. al., 2012) e (GODOWITCH et. al., 2010).

Diversos fatores podem influenciar a formação de $\mathrm{NO}_{\mathrm{x}}$, entre eles destacam-se: a temperatura de combustão, a velocidade de fluxo no local da combustão, a composição do combustível e a taxa de dosagem de combustível/ar (SARAVANAN et. al., 2012) e (BAUKAL, 2004).

$O$ aumento da presença de compostos gasosos nitrogenados determina um estado de alerta não somente pela sua toxicidade (LAWRENCE et. al., 2005) e (BLONDEAU et. al., 2005), mas também por serem espécies precursoras fundamentais que estão intrinsecamente envolvidas na produção fotoquímica de ozônio troposférico, outro sério poluente tóxico e partículas finas (GODOWITCH et. al., 2010). Além disso, o $\mathrm{NO}_{x}$ é também um dos principais terminadores das reações envolvendo radicais livres na atmosfera (SUN et. al., 2011), (RAIVONEN et. al., 2009), (PENGA et. al., 2006), (CARL et. al., 2001), (CHAMEIDES et. al., 1988), (GUANGFENG et. al., 2004) e (CASSANDRA et. al., 2006).

Estudos realizados até hoje ainda não demonstraram o perigo à saúde que pode ser causado pelo monóxido de nitrogênio (NO) nas concentrações encontradas nas grandes cidades. $\mathrm{Na}$ verdade, sabe-se que o grande risco deste composto na atmosfera está associado em dias de grande radiação, ao fato do mesmo ser rapidamente oxidado formando dióxido de nitrogênio $\left(\mathrm{NO}_{2}\right)$, conforme a Equação 1.

$$
2 \mathrm{NO}+\mathrm{O}_{2} \rightarrow 2 \mathrm{NO}_{2}
$$

equação (1)

De acordo com a Organização Mundial de Saúde (WHO), o $\mathrm{NO}_{2}$ é o mais tóxico dos dois gases. $\mathrm{O} \mathrm{NO}$ é um gás incolor e que contém um odor característico (WHO, 2006). O dióxido de nitrogênio é um poluente que causa irritabilidade aos olhos, nariz e mucosas. Além desses problemas de irritabilidade, $\mathrm{o} \mathrm{NO}_{2}$ provoca uma série de graves doenças respiratórias como enfisema pulmonar, bronquites, traqueítes e em casos mais graves, câncer.

Estudos toxicológicos recentes relatam efeitos agudos após exposição a altas concentrações de $\mathrm{NO}_{2}$ por 1 hora, chegando a um valor de referência adotado pela OMS de 200 $\mu \mathrm{g} / \mathrm{m}^{3}$. Devido aos efeitos adversos causados pela exposição a poluentes atmosféricos no ar sobre os seres humanos, a União Europeia também estabeleceu normas para os poluentes no ar. Segundo a Agência Europeia do Meio Ambiente (EEA), as concentrações toleradas para o dióxido de nitrogênio também são da ordem de $200 \mu \mathrm{g} / \mathrm{m}^{3}$ para 1 hora de exposição (EEA, 2012).

A USEPA (Agência de Proteção ao Meio Ambiente dos Estados Unidos) indica dois tipos de padrões de qualidade do ar ambiente: os padrões primários, que fornecem proteção à saúde pública, incluindo a proteção à saúde das populações "sensíveis", como os asmáticos, crianças e idosos e os padrões secundários, que fornecem proteção ao bem-estar público. O padrão 
estabelecido pelo órgão norte americano para o $\mathrm{NO}_{2}$ é de 100 ppb em 1 hora de exposição (EPA, 2012).

No Brasil destaca-se a resolução CONAMA 03 de 1990 que apresenta como padrão

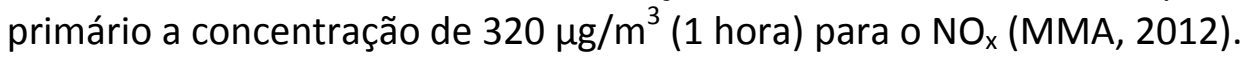

Na Tabela 1 são apresentados dados dos padrões de qualidade do ar estabelecidos pelas principais agências mundiais de proteção ao meio ambiente.

Tabela 1 - Principais padrões de qualidade do ar do Brasil e do mundo.

\begin{tabular}{c|c|c}
\hline LEGISLAÇÃO & TEMPO DE EXPOSIÇÃO & CONCENTRAÇÃO \\
\hline OMS & 1 hora & $200 \mu \mathrm{g} / \mathrm{m}^{3}$ \\
\hline EEA & 1 hora & $200 \mu \mathrm{g} / \mathrm{m}^{3}$ \\
\hline USEPA & 1 hora & $100 \mathrm{ppb}$ \\
\hline CONAMA & 1 hora & $320 \mu \mathrm{g} / \mathrm{m}^{3}$ \\
\hline
\end{tabular}

Estudos realizados por Vieira et al. (2012) em crianças da zona urbana de São Paulo, Brasil, mostram que os níveis de $\mathrm{NO}_{2}$ presentes no ar provocam sintomas tipicamente associados a doenças respiratórias. Toda a população estudada apresentou sibilo, ruído semelhante a um assobio agudo característico da asma, em alguma etapa do estudo realizado. Neste estudo constatou-se que $45,8 \%$ dos casos tiveram uma evolução para o diagnóstico de asma.

\subsection{Dióxidos de Enxofre $\left(\mathrm{SO}_{2}\right)$}

Muitos países têm experimentado um rápido desenvolvimento econômico e urbano nas últimas décadas, alterando os níveis de $\mathrm{SO}_{2}$ em suas grandes cidades (PAN et. al., 2010). O enxofre presente na atmosfera, tanto na forma de gases quanto na forma de aerossóis, causa um grande impacto no meio ambiente. Possíveis alterações climáticas, bem como danos a saúde de vários organismos podem estar associados à elevada concentração de tal poluente (EISINGER e BURROWS, 1998), (YANG et. al., 2009) e (KROTKOV et. al., 2006).

Vários estudos têm mostrado a correlação entre concentração de $\mathrm{SO}_{2}$ e os parâmetros meteorológicos (LUVSANA et. al., 2012), (AKPINAR et. al., 2008), (BRIDGMAN et. al., 2002), (ILIC et. al., 2010) e (WANG et. al., 2011). O dióxido de enxofre entra na atmosfera através de uma série de atividades antrópicas e fenômenos naturais. Grandes quantidades são lançadas diretamente na troposfera, resultado da queima de combustíveis fósseis, e em certa medida pela oxidação da matéria orgânica no solo, pela oxidação de $\mathrm{H}_{2} \mathrm{~S}$ sobre oceanos, pelas erupções vulcânicas e pela queima de biomassa (EISINGER e BURROWS, 1998).

O dióxido de enxofre possui uma alta reatividade com os outros poluentes da atmosfera (WANG et. al., 2002). Além disso, $\mathrm{o} \mathrm{SO}_{2}$ é um dos principais precursores da chuva ácida (INOMATA et. al., 2006) e também pode ser considerado como um grande gerador de outras formas poluentes.

Segundo Kulmala et al. (2004), o gás $\mathrm{SO}_{2}$ é o principal precursor de novas partículas na atmosfera aumentando a exposição humana a partículas ultrafinas. $\mathrm{O} \mathrm{SO}_{2}$ ao ser oxidado na atmosfera forma o aerossol de sulfato, um dos principais componentes das partículas finas em suspensão (VERHEGGEN et. al., 2002). 
Estudos epidemiológicos revelam uma associação entre a poluição do ar e o alto nível de morbidade e mortalidade por doenças respiratórias e câncer de pulmão (PAN et. al., 2010). Estudos controlados indicam mudanças na função pulmonar e sintomas respiratórios após períodos curtos de exposição ao $\mathrm{SO}_{2}$. Com base nesta evidência, recomenda-se que uma concentração de $\mathrm{SO}_{2}$ de $500 \mu \mathrm{g} / \mathrm{m}^{3}$ não deve ser excedida durante uma média de períodos de 10 minutos de duração. Já para exposições em longo prazo, a indicação fica entorno de $20 \mu \mathrm{g} / \mathrm{m}^{3} \mathrm{em}$ uma média de 24 horas (XIAOLIN et. al., 2009).

Enquanto a USEPA recomenda valores de $75 \mathrm{ppb}$ e $0,5 \mathrm{ppb}$ para os padrões primários e secundários respectivamente. A legislação brasileira, por sua vez, é menos exigente recomendando uma concentração média anual de $80 \mu \mathrm{g} / \mathrm{m}^{3}$ de ar e uma concentração média de 24 horas de $365 \mu \mathrm{g} / \mathrm{m}^{3}$ de ar, não podendo a mesma ser excedida mais de uma vez por ano.

\subsection{Material Particulado}

O material particulado (MP) abrange uma grande quantidade de poluentes, que devido ao pequeno tamanho, encontram-se suspensos na atmosfera. O material particulado está representado pelos materiais totais em suspensão (PTS), partículas com diâmetro igual ou superior $100 \mu \mathrm{m}$ e materiais com diâmetros de $10 \mu \mathrm{m}$ e 2,5 $\mu \mathrm{m}\left(\mathrm{PM}_{10}\right.$ e $\left.\mathrm{PM}_{2,5}\right)$. O PTS somado com o $\mathrm{PM}_{10}$ e $\mathrm{PM}_{2,5}$ formam o material particulado conhecido na comunidade científica (COELHO, 2007).

As partículas finas têm um tempo de residência longo na atmosfera e podem penetrar profundamente nos pulmões, assim, a toxicidade do material particulado fino é mais elevada do que a de partículas grossas no ar (PACYNA, 1995).

O MP é uma mistura heterogênea e complexa constituída por partículas sólidas e líquidas capaz de transportar uma grande variedade de compostos químicos prejudiciais à saúde humana (TRAVERSI, 2009). As partículas primárias são as emitidas diretamente na atmosfera e as partículas secundárias são produzidas a partir de reações químicas entre os precursores de gases atmosféricos ou entre estes e as partículas primárias (WHO, 2005).

No inicio dos estudos, considerava-se somente as partículas em suspensão (PTS), partículas que continham o tamanho menor que $50 \mu \mathrm{m}$. No entanto, pesquisas mostraram que as partículas finas são as maiores causadoras de danos a saúde, pelo fato de terem a maior capacidade de penetração no aparelho respiratório.

Dentro do universo das partículas finas, as que mais chamam atenção em função dos problemas de saúde são as partículas com tamanhos de $10 \mu \mathrm{m}$ e 2,5 $\mu \mathrm{m}, \mathrm{PM}_{10}$ e $\mathrm{PM}_{2,5}$ respectivamente. Em destaque, a fração de material particulado $2,5 \mu \mathrm{m}$ é capaz de ultrapassar a região alveolar dos pulmões. Estas propriedades físicas associadas às características químicas explicam as propriedades perigosas do $\mathrm{MP}_{2,5}$ à saúde humana (TRAVERSI, 2009), (BRUGGE et. al., 2007) e (CIENCEWICKI et. al., 2008).

As partículas finas $\left(\mathrm{PM}_{2,5}\right)$ são originadas por fontes naturais e antropogênicas. As fontes naturais são atribuídas à poeira do solo, ao sal do mar, aos distúrbios geológicos, aos restos biológicos, aos incêndios florestais e a oxidação de gases biogênicos reativos, enquanto as partículas das fontes antropogênicas são decorrentes da queima de combustíveis fósseis por 
fontes fixas e móveis, as emissões fugitivas, e várias atividades industriais, comerciais e residenciais (CHAN e YAO, 2008) e (VIANA et. al., 2008).

A exposição por longo prazo a altas concentrações de material particulado pode aumentar o risco de câncer no pulmão, doenças respiratórias obstrutivas crónicas (COPD), e arteriosclerose, ao passo que a exposição de curto prazo pode causar exacerbação de outras doenças respiratórias, incluindo bronquite e asma, bem como mudanças na variabilidade da frequência cardíaca (BRUNEKREEF e HOLGATE, 2002), (DOMINICl et. al., 2007), (ELLIOTT et. al., 2007), (HALES e HOWDEN-CHAPMAN, 2007), (POPE III, 2004), (SAMET et. al., 2000) e (SORENSEN et. al., 2003).

Segundo a Organização Mundial de Saúde, as $\mathrm{MP}_{2,5}$ provocam efeitos adversos para a saúde a nível de sistema respiratório e cardiovascular, sendo toda a população afetada. Em comum, a susceptibilidade a poluição das partículas de $2,5 \mu \mathrm{m}$ varia com a idade e estado de saúde.

Na Tabela 2 são apresentados os padrões de qualidade recomendado pela Agência Europeia do Ambiente, pela Agência Nacional de Proteção ao Meio Ambiente dos EUA e Conselho Nacional do Meio Ambiente.

Tabela 2 - Padrões de qualidade do ar para o poluente os materiais particulados segundo a EEA, USEPA e CONAMA.

\begin{tabular}{|c|c|c|c|c|}
\hline & POLUENTE & PADRÕES & CONCENTRAÇÃO & TEMPO DE EXPOSIÇÃO \\
\hline \multirow[t]{2}{*}{ EEA } & $\mathrm{PM}_{2,5}$ & - & $25 \mu \mathrm{g} / \mathrm{m}^{3}$ & 1 ano \\
\hline & $\mathrm{PM}_{10}$ & - & $50 \mu \mathrm{g} / \mathrm{m}^{3}$ & 24 horas \\
\hline \multirow[t]{4}{*}{ USEPA } & \multirow[t]{3}{*}{$\mathrm{PM}_{2,5}$} & Primário & $12 \mu \mathrm{g} / \mathrm{m}^{3}$ & Anual \\
\hline & & Secundário & $15 \mu \mathrm{g} / \mathrm{m}^{3}$ & Anual \\
\hline & & $\begin{array}{l}\text { Primário e } \\
\text { Secundário }\end{array}$ & $35 \mu \mathrm{g} / \mathrm{m}^{3}$ & 24 horas \\
\hline & $\mathrm{PM}_{10}$ & $\begin{array}{l}\text { Primário e } \\
\text { Secundário }\end{array}$ & $150 \mu \mathrm{g} / \mathrm{m}^{3}$ & 24 horas \\
\hline \multirow[t]{2}{*}{ CONAMA } & \multirow[t]{2}{*}{$\mathrm{PM}_{2,5}+\mathrm{PM}_{10}$} & \multirow{2}{*}{$\begin{array}{l}\text { Primário e } \\
\text { Secundário }\end{array}$} & $50 \mu \mathrm{g} / \mathrm{m}^{3}$ & 1 ano \\
\hline & & & $150 \mu \mathrm{g} / \mathrm{m}^{3}$ & 24 horas \\
\hline
\end{tabular}

Levando em consideração os "guidelines" da OMS os padrões de qualidade do ar recomendados para os materiais particulados são: $\mathrm{PM}_{2,5}: 10 \mu \mathrm{g} / \mathrm{m}^{3}$ (média anual) e $25 \mu \mathrm{g} / \mathrm{m}^{3}$ (24 h); $\mathrm{PM}_{10}: 20 \mu \mathrm{g} / \mathrm{m}^{3}$ (média anual) e $50 \mu \mathrm{g} / \mathrm{m}^{3}$ (24 h) (PACYNA, 1995).

A legislação brasileira recomenda os valores de padrão primário e secundário às concentrações médias anuais de 50 (cinquenta) microgramas por metro cúbico de ar e a concentração média de 24 (vinte e quatro) horas de 150 (cento e cinquenta) microgramas por metro cúbico de ar, que não devendo ser excedida mais de uma vez por ano.

\section{POLUENTES ESPECÍFICOS}

O termo Compostos Orgânicos Voláteis (COVs) engloba um grande grupo de poluentes que se encontram presentes no ar atmosférico, principalmente, sob a forma gasosa. Mais de 500 compostos têm sido classificados como compostos orgânicos voláteis (HOSHI et. al., 2008). 
$\mathrm{Na}$ maioria das classificações aceitas geralmente, os COVs são baseados nas suas propriedades físico-químicas como, por exemplo, o ponto de ebulição e a pressão de vapor (KRÓL et. al., 2010). Para a OMS, o termo, "compostos orgânicos voláteis" deve ser utilizado apenas para compostos adsorvidos em um adsorvente sólido e com ponto de ebulição entre 50 e $260{ }^{\circ} \mathrm{C}$ (KRÓL et. al., 2010). Em contrapartida, a definição da USEPA inclui compostos polares e não polares de $C_{2}$ a $C_{10}$, cuja pressão de vapor a $25{ }^{\circ} \mathrm{C}$ seja superior a $13,33 \mathrm{~Pa}$ (WANG e AUSTIN, 2006).

Nas Tabelas 3 e 4 os COVs são subdivididos e classificados de acordo com o grau de volatilidade e em relação à sua ação sobre os ecossistemas e saúde humana.

Tabela 3 - Classificação para os compostos orgânicos pelo grau de volatilidade.

\begin{tabular}{c|c|c}
\hline NOME & ABREVIATURA & PONTO DE EBULIÇÃO $\left({ }^{\circ} \mathrm{C}\right)$ \\
\hline Compostos Orgânicos Muito Voláteis & COMVs & $<0-100$ \\
\hline Compostos Orgânicos Voláteis & COVs & $50-100$ a 240 - 260 \\
\hline Compostos Orgânicos Semi-voláteis & COSVs & $240-260$ a $380-400$ \\
\hline $\begin{array}{c}\text { Compostos Orgânicos Não-voláteis } \\
\text { associados a material particulado }\end{array}$ & MOP & $>380$ \\
\hline
\end{tabular}

Adaptado de Król, S. et al. (2010).

Tabela 4 - Classificação de compostos orgânicos voláteis (COV) em relação à sua ação sobre os ecossistemas e a saúde humana.

\begin{tabular}{|c|c|c|}
\hline ABREVIATURA & CARACTERÍSTICAS & EXEMPLOS \\
\hline COV-OX & $\begin{array}{c}\text { Alto potencial para a geração de oxidantes } \\
\text { fotoquímicos }\left(\mathrm{O}_{3}, \mathrm{PAN}\right)\end{array}$ & $\begin{array}{c}\text { Alcanos } C_{2}-C_{8} \\
\text { Alcenos } C_{2}-C_{8} \\
\text { Alcinos } C_{2}-C_{8} \\
\text { Xilenos } \\
\text { Tetrametilbenzenos }\end{array}$ \\
\hline COV-TOX & Altamente tóxico para os organismos vivos & $\begin{array}{l}\text { Hidrocarbonetos clorados } \\
\text { Hidrocarbonetos oxigenados }\end{array}$ \\
\hline COSVs & $\begin{array}{l}\text { Prejudicam a saúde humana (lesões na pele, } \\
\text { alergias) }\end{array}$ & $\begin{array}{l}\text { PCB } \\
\text { PAH } \\
\end{array}$ \\
\hline COV-FORM & $\begin{array}{c}\text { Prejudicam a saúde humana (lesões na pele, } \\
\text { alergias) }\end{array}$ & $\begin{array}{c}\text { Ácidos graxos livres } \\
\text { Aldeídos } \\
\text { Ésteres } \\
\text { Cetonas }\end{array}$ \\
\hline COV-STRAT & $\begin{array}{c}\text { Altamente capaz de destruir a camada de } \\
\text { ozônio estratosférico }\end{array}$ & $\begin{array}{c}\text { Clorofluorocarbonos } \\
\text { Halocarbonos } \\
\text { Hidrocarbonetos Clorados }\end{array}$ \\
\hline COV-CLAIM & Elevado potencial de aquecimento global & $\begin{array}{c}\text { DMS } \\
\text { Metano }\end{array}$ \\
\hline
\end{tabular}

Adaptado de Król, S. et al. (2010).

A presença e a composição de COVs na atmosfera urbana estão intimamente relacionadas com o uso de combustíveis veiculares (MARTINS et al., 2007). Os compostos carbonílicos e os hidrocarbonetos aromáticos estão entre os principais poluentes orgânicos no ar urbano (JONES, 1999). 
Os COVs têm sido conhecidos por degradar a saúde humana (WHO, 2005). Nos últimos anos, muitos estudos relacionados à qualidade do ar foram realizados tendo como foco principal os seguintes compostos: benzeno, tolueno, etilbenzeno e xilenos, conhecidos no meio científico por BTEX (AO et al., 2004), (AO e LEE, 2002) e (DUTTA et al., 2009).

O benzeno, o etilbenzeno, o tolueno e os xilenos, representam uma fração significativa dos compostos orgânicos voláteis emitidos em atmosferas urbanas (SINGH et al., 1992), (BAILEY e EGGLESTON, 1993) e (ZIELINSKA et al., 1996). As principais fontes geradoras de BTEX são os veículos, as estações de autosserviço móvel e as emissões industriais. No caso das emissões veiculares existem várias contribuições, principalmente relacionadas às emissões de escape (fria e quente), as emissões por evaporação e as emissões de freio e de desgaste dos pneus (CASELLI et. al., 2010).

Para estimar os riscos à saúde humana, os poluentes normalmente são classificados como cancerígenos e não cancerígenos. Os BTEX, principalmente o benzeno, são apontados como compostos carcinogênicos, que veem degenerando a saúde humana, principalmente, nas grandes cidades (JONES, 1999), (CRUMP, 1994) e (BÁEZ et. al., 2003).

A OMS propõe orientações para os BTEX, mas estas não são recomendações compulsórias. A USEPA não propõe quaisquer padrões para o benzeno, tolueno e outros COVs no ambiente. Em contra partida, a Agência de Segurança e Saúde Ocupacional (OSHA) dos Estados Unidos define alguns padrões, conforme mostrados nas Tabelas 5 e 6 (OSHA, ano) e (HAN e NAEHER, 2006).

Tabela 5 - Valores regulamentares determinados pela OSHA.

\begin{tabular}{c|c|c}
\hline POLUENTES & EXPOSIÇÃO & VALORES \\
\hline Benzeno & $\begin{array}{c}\text { 8-h dia de trabalho ou } \\
\text { 40-h semana de trabalho }\end{array}$ & $\begin{array}{c}1 \mathrm{ppm} \\
3,19 \mathrm{mg} / \mathrm{m}^{3}\end{array}$ \\
\hline Tolueno & $\begin{array}{c}\text { 8-h dia de trabalho ou } \\
\text { 40-h semana de trabalho }\end{array}$ & $\begin{array}{c}200 \mathrm{ppm} \\
753,6 \mathrm{mg} / \mathrm{m}^{3}\end{array}$ \\
\hline Etilbenzeno & 8-h dia de trabalho ou & $100 \mathrm{ppm}$ \\
& 40-h semana de trabalho & $434 \mathrm{mg} / \mathrm{m}^{3}$ \\
\hline Xileno & 8-h dia de trabalho ou & $100 \mathrm{ppm}$ \\
& 40-h semana de trabalho & $434 \mathrm{mg} / \mathrm{m}^{3}$ \\
\hline
\end{tabular}

Definitivamente, a preocupação não é somente com o ar externo, o ar atmosférico, mas também com o ar no interior do veículo. A OMS já admite o que a poluição no interior do veículo, uma grande ameaça à saúde (CHEN et al., 2011). Em um estudo no Japão foram identificados 162 compostos orgânicos na cabine de um carro novo (TOSHIAKI e ICHIRO, 2006).

No Brasil, o Ministério do Trabalho e Emprego (MTE, 1978) estabelece valores de exposição para ambientes de trabalho conforme a NR-15, anexos no 11 e 13-A, no que se refere aos agentes químicos e atividades e operações insalubres para o benzeno, tolueno, etilbenzeno e xilenos.

O benzeno pelo fato de ser o mais agressivo dos compostos citados é tratado de forma separada no anexo 13-A da NR-15, de forma tal que os valores referenciados não excluem os riscos a saúde, mas pressupõe a menor concentração possível de acordo com a exequibilidade técnica, uma vez que para o benzeno, classificado como um produto cancerígeno, não possui 
limite seguro de exposição. Já as concentrações de tolueno, etilbenzeno e de xilenos totais devem ser consideradas de risco grave e iminente quando ultrapassarem os valores apresentados no anexo 11 da NR-15 (BONETTI, 2011), conforme a Tabela 6.

Tabela 6 - Tabela de limites de tolerância para benzeno, tolueno, etilbenzeno e xilenos e seus isômeros.

\begin{tabular}{c|c|c}
\hline POLUENTES & EXPOSIÇÃO & RECOMENDAÇÕES \\
\hline Benzeno & $48 \mathrm{~h} / \mathrm{semana}$ & $3,19 \mathrm{mg} / \mathrm{m}^{3}$ \\
\hline Tolueno & $48 \mathrm{~h} /$ semana & $290 \mathrm{mg} / \mathrm{m}^{3}$ \\
\hline Etilbenzeno & $48 \mathrm{~h} /$ semana & $340 \mathrm{mg} / \mathrm{m}^{3}$ \\
\hline Xileno & $48 \mathrm{~h} /$ semana & $340 \mathrm{mg} / \mathrm{m}^{3}$ \\
\hline \multicolumn{2}{|c}{ Fonte: MTE (1978). }
\end{tabular}

Hinwood et al. (2006) realizaram um estudo em 32 locais na Austrália, entre veículos motorizados e em pequenos locais ao ar livre. 26 compostos orgânicos foram identificados e dentre eles, 10 foram eram encontrados mais comumente. Os dez compostos citados foram representados pelas moléculas do benzeno, Clorometano, tolueno, etilbenzeno, xileno, 1,3 butadieno, clorofórmio, diclorometano, 1,1,2-tricloro-1,2,2-trifluoroetano e Tetracloroetileno.

Tabela 7 - Concentração dos COVs (ppb) mais comuns nas amostras coletadas na Austrália.

\begin{tabular}{|c|c|c|c|c|c|c|c|c|c|c|}
\hline Locais & Benzeno & Tolueno & $\begin{array}{c}\text { Etilben- } \\
\text { zeno }\end{array}$ & Xileno & $\begin{array}{c}\text { 1,3- } \\
\text { Butadieno }\end{array}$ & $\begin{array}{l}\text { Clorome- } \\
\text { tano }\end{array}$ & $\begin{array}{l}\text { Clorofór- } \\
\text { mio }\end{array}$ & $\begin{array}{l}\text { Dicloro- } \\
\text { metano }\end{array}$ & $\begin{array}{c}\text { 1,1,2-Tricloro- } \\
1,2,2- \\
\text { trifluoroetano }\end{array}$ & $\begin{array}{c}\text { Tetracloro } \\
\text {-etileno }\end{array}$ \\
\hline $\begin{array}{c}\text { Estaciona } \\
\text { mento } \\
\text { coberto }\end{array}$ & 5,6 & 24,7 & 2 & 13,4 & 0,4 & 0,2 & 0,1 & bdl & bdl & bdl \\
\hline $\begin{array}{c}\text { Estaciona } \\
\text { mento 40 } \\
\text { andar }\end{array}$ & 3,1 & 9,6 & 1,1 & 6,1 & 0,6 & bdl & bdl & bdl & bdl & bdl \\
\hline $\begin{array}{c}\text { Clube } \\
\text { noturno }\end{array}$ & 4,5 & 11,5 & 4,7 & 29,4 & 3,5 & 3,7 & 6,7 & bdl & 0,4 & 0,6 \\
\hline $\begin{array}{l}\text { Interior de } \\
\text { residência } \\
\text { com } \\
\text { garagem }\end{array}$ & 0,7 & 3,5 & 0,4 & 2,2 & bdl & 0,7 & bdl & bdl & bdl & bdl \\
\hline $\begin{array}{l}\text { Interior de } \\
\text { residência } \\
\text { (dia) }\end{array}$ & 0,3 & 0,5 & 0,1 & 0,4 & Bdl & 0,7 & bdl & bdl & bdl & bdl \\
\hline $\begin{array}{l}\text { Shopping } \\
\text { Center }\end{array}$ & 1,7 & 4,1 & 0,2 & 1,5 & bdl & 3,9 & 0,5 & bdl & bdl & bdl \\
\hline $\begin{array}{l}\text { Interior de } \\
\text { escritório }\end{array}$ & 1,1 & 2,7 & bdl & 1 & bdl & bdl & bdl & bdl & bdl & bdl \\
\hline $\begin{array}{c}\text { Área } \\
\text { Industrial }\end{array}$ & 2,1 & 4,4 & 0,2 & 1,7 & bdl & bdl & bdl & bdl & bdl & bdl \\
\hline
\end{tabular}

Fonte: Hinwood et al. (2006) bdl: amostras abaixo do limite de detecção.

Zhang et al. (2012) mediram os níveis de BTEX no ar ambiente de Pequim, na China. Os autores realizaram amostragem durante dois anos consecutivos em todas as estações do ano e encontraram uma concentração de BTEX média de: $31,9 \mu \mathrm{g} / \mathrm{m}^{3}$ no inverno; $27,2 \mathrm{mg} / \mathrm{m}^{3}$ no outono; $23,2 \mu \mathrm{g} / \mathrm{m}^{3}$ na primavera e $19,1 \mu \mathrm{g} / \mathrm{m}^{3}$ no verão. 


\section{CONCLUSÃO}

O monitoramento da qualidade do ar é uma importante ferramenta de avaliação da poluição em atmosferas urbanas e industriais, principalmente em função dos riscos ao meio ambiente e à saúde apresentados por poluentes como $\mathrm{NO}_{x}, \mathrm{SO}_{2}$, materiais particulados, $\mathrm{O}_{3}$, metais e orgânicos (BTEX, clorados, pesticidas, etc). Oportunamente, estudos são necessários para elucidar as causas, níveis e consequências desta poluição visando prioritariamente o controle das emissões poluentes e a melhoria da qualidade de vida das populações expostas.

\section{AGRADECIMENTO}

A FUNCAP, pelo financiamento (bolsa de estudo) durante o período da pesquisa.

\section{REFERÊNCIAS BIBLIOGRÁFICAS}

1. Agencia de Proteção Ambiental <http://www.epa.gov/air/criteria.html> acessado em: 30/12/1012.

2. Agência Europeia do Meio Ambiente <http://ec.europa.eu/environment/air/quality/standards.htm> acessado em: 30/12/1012.

3. AKPINAR, S.; OZTOP, H.; KAVAK AKPINAR, E. Evaluation of relationship between meteorological parameters and air pollutant concentrations during winter season in Elazig, Turkey. Environmental Monitoring and Assessment, v.146, n.1 - 3, p.211 - 224, 2008.

4. AO, C.H.; LEE, S.C. Removal of indoor air ppb level volatile organic compounds (VOCs) and NOx by heterogeneous photocatalysis better air quality in asian and pacific rim cities (BAQ 2002). 16 - 18 de dezembro de 2002, Hong Kong SAR. Disponível em: <http://www.epd.gov.hk/epd/english/news events/events/baq2002.html> Acesso em: 09/05/2013.

5. AO, C.H.; LEE, S.C.; ZOU, S.C.; MAK, C.L. Inhibition effect of $\mathrm{SO}_{2}$ on $\mathrm{NO}_{x}$ and VOCs during the photodegradation of synchronous indoor air pollutants at parts per billion (ppb) level by $\mathrm{TiO}_{2}$. Applied Catalysis B: Environmental, v.49, n.3, p.187 - 193, 2004.

6. BÁEZ, A.P.; PADILLAH, H.; GARCÍA R.; TORRES M.C.; ROSAS, I.; BELMONT, R. Carbonyl levels in indoor and outdoor air in Mexico city and Xalapa, Mexico. Science of the Total Environment, v.302, n.1-3, p.211 - 226, 2003.

7. BAILEY, J.C.; EGGLESTON, S. The contribution of gasoline fuelled vehicle exhaust to the UK speciated hydrocarbon inventory. Science of Total Environment, v.134, n.1-3, p.263 - 271, 1993.

8. BAUKAL, C.E. Industrial Combustion Pollution and Control, Marcel Dekker Inc., New York, 2004, p.247 - 325.

9. BLONDEAU, P.; IORDACHE, V.; POUPARD, O.; GENIN, D.; ALLARD, F. Relationship between outdoor and indoor air quality in eight french schools. Indoor Air, v.15, n.1, p.2 - 12, 2005. 
10. BONETTI, T.M. Desenvolvimento de metodologia analítica para a avaliação de contaminações atmosféricas por BTEX em postos de abastecimento de combustíveis. 2011. 155 f. Dissertação (Mestrado) - Programa de Pós-Graduação em Engenharia Química, Universidade Federal de Santa Catarina, Centro Tecnológico, Florianópolis, 2011.

11. BRASIL. Ministério do Meio Ambiente, Conselho Nacional do Meio Ambiente, CONAMA. Resolução CONAMA no 003/90, de 28 de junho de 1990. Disponível em: <http://www.mma.gov.br> Acesso em: 30/12/2012.

12. BRASIL. Ministério do Trabalho e do Emprego-MTE. N-15 - Atividades e Operações Insalubres, 6 Julho de 1978. Disponível em: <http://portal.mte.gov.br> Acessado em: 10/05/2013.

13. BRIDGMAN, H.A.; DAVIES, T.D.; JICKELLS, T.; HUNOVA, I.; TOVEY, K.; BRIDGES, K.; SURAPIPITH, V. Air pollution in the Krusne Hory region, Czech Republic during the 1990. Atmospheric Environment, v.36, n.21, p.3375 - 3389, 2002.

14. BRUGGE, D.; DURANT. J.L.; RIOUX, C. Near-highway pollutants in motor vehicle exhaust: a review of epidemiologic evidence of cardiac and pulmonary health risks. Environ. Health, v.6, n.1, p.23 - 35, 2007.

15. BRUNEKREEF, B.; HOLGATE, S.T. Air pollution and health. The lancet, v.360, n.9341, p.1233 1242, 2002.

16. CARL, M.; BERKOWITZ, R.A.; BIAN, Z.X.; ZHONG, S.; ROBERT, S.; DISSELKAMP, N.S.; LAULAINEN, E.G.C. Aircraft observations of aerosols, $\mathrm{O}_{3}$ and $\mathrm{NO}_{\mathrm{y}}$ in a night time urban plume. Atmospheric Environment, v.35, n.13, p.2395 - 2404, 2001.

17. CASELLI, M.; DE GENNARO, G.; MARZOCCA, A.; TRIZIO, L.; TUTINO, M. Assessment of the impact of the vehicular traffic on BTEX concentration in ring roads in urban areas of Bari (Italy). Chemosphere, v.81, n.3, p.306 - 311, 2010.

18. CASSANDRA, V.H.; MUNGER, J.W.; STEVEN, C.W.; ZAHNISER, M.; NELSON, D.J.; MCMANUS, B. Atmospheric reactive nitrogen concentration and flux budgets at a Northeastern US forest site. Agric. for Meteorol., v.136, n.1-4, p.159 - 174, 2006.

19. CHAMEIDES, W.L.; LINDSAY, R.W.; RICHARDSON, J. KIANG, C.S., The role of biogenic hydrocarbons in urban photochemical smog: Atlanta as case study. Science, v.241, n.4872, p.1473 - 1475, 1988.

20. CHAN, C.K., YAO, X. Air pollution in mega cities in China. Atmospheric Environment, v.42, n.1, p.1 - 42, 2008.

21. CHEN, X.; ZHANG, G.; ZHANG, Q.; CHEN, H. Mass concentrations of BTEX inside air environment of buses in Changsha, China. Building and Environment, v.46, n.2, p.421 - 427, 2011.

22. CIENCEWICKI, J.; TRIVEDI, S.; KLEEBERGER, S.R. Oxidants and the pathogenesis of lung diseases. J. Allergy Clin. Immun., v.122, n.3, p.456 - 68, 2008.

23. COELHO, M.S.Z.S. Uma análise estatística com vistas a previsibilidade de doenças respiratórias em função de condições meteorotrópicas na cidade de São Paulo no ano de 2007. 202 f. Tese (Doutorado em Ciências) - Departamento de Ciências Atmosféricas, Universidade de São Paulo, São Paulo, 2007. 
24. CRUMP K.S. Risk of benzene-induced leukemia: A sensitivity analysis of the pliofilm cohort with additional follow-up and new exposure estimates. Environmental Health, v.42, n.2, p.219 - 242, 1994.

25. DOMINICI, F.; PENG, R.D.; ZEGER, S.L.; WHITE, R.H.; SAMET, J.M. Particulate air pollution and mortality in the United States: did the risks change from 1987 to 2000 . American Journal of Epidemiology, v.166, n.8, p.880 - 888, 2007.

26. DUTTA, C., SOM, D.; CHATTERJEE, A.; MUKHERJEE, A.K.; JANA, T.K.; SEN, S. Mixing ratios of carbonyls and BTEX in ambient air of Kolkata, India and their associated health risk. Environmental Monitoring and Assessment, v.148, n.1 - 4, p.97 - 107, 2009.

27. EISINGER, M. e BURROWS, J. Tropospheric sulfur dioxide observed by the ERS-2 GOME instrument. Geophysical Research Letters, v.25, n.22, p.4177 - 4180, 1998.

28. ELLIOTT, P.; SHADDICK, G.; WAKEFIELD, J.C.; DE HOOGH, C.; BRIGGS, D.J. Long term associations of outdoor air pollution with mortality in Great Britain. Thorax, v.62, n.12, p.1088 - 1094, 2007.

29. GODOWITCH, J.M.; POULIOT, G.A.; RAO, S.T. Assessing multi-year changes in modeled and observed urban $\mathrm{NO}_{x}$ concentrations from a dynamic model evaluation perspective. Atmospheric Environment, v.44, n.24, p.2894 - 2901, 2010.

30. GUANGFENG, J., JEROME, D.F. Modeling the effects of VOCs and $\mathrm{NO}_{\mathrm{x}}$ emission sources on ozone formation in Houston during the TexAQS 2000 field campaign. Atmospheric Environment, v.38, n.30, p.5071 - 5085, 2004.

31. HALES, S.; HOWDEN-CHAPMAN, P. Effects of air pollution on health. BMJ, v.335, n. 7615, p.314 - 315, 2007.

32. HAN, X. e NAEHER, L.P.A Review of traffic-related air pollution exposure assessment studies in the developing world. Environment International, v.32, n.1, p.106 - 120, 2006.

33. HINWOOD, A.L.; BERKO, H.N.; FARRAR, D.; GALBALLY, I.E.; WEEKS, I.A. Volatile organic compounds in selected micro-environments. Chemosphere, v.63, p.421 - 429, 2006.

34. HORGNIES, M.; DUBOIS-BRUGGER, I.; GARTNER, E.M. NO $\mathrm{N}_{\mathrm{x}}$ de-pollution by hardened concrete and the influence of activated charcoal additions. Cement and Concrete Research, v.42, p.1348 - 1355, 2012.

35. HOSHI, J.; AMANO, S.; SASAKI, Y.; KORENAGA, T. Investigation and estimation of emission sources of 54 volatile organic compounds in ambient air in Tokyo. Atmospheric Environment, v.42, n.10, p.2383 - 2393, 2008.

36. ILIC, I.Z.; ZIVKOVIC, D.T.; VUSOVIC, N.M.; BOGDANOVIC, D.M. Investigation of the correlation dependence between $\mathrm{SO}_{2}$ emission concentration and meteorological parameters: case study DBor (Serbia). Journal of Environmental Science and Health, Part A, v.45, p.901 - 907, 2010.

37. INOMATA, Y.; IWASAKA, Y.; OSADA, K.; HAYASHI, M.; MORI, I.; KIDO, M.; HARA, K.; SAKAI, T. Vertical distributions of particles and sulfur gases (volatile sulfur compounds and $\mathrm{SO}_{2}$ ) over east Asia: comparison with two aircraft borne measurements under the asian continental out flow in spring and winter. Atmospheric Environment, v.40, n.3, p.430 - 444, 2006.

38. JONES, A.P. Indoor air quality and health. Atmospheric Environment, v. 33, n. 28, p. 4535 4564, 1999. 
39. KRÓL, S.; ZABIEGAŁA, B.; NAMIESNIK, J. Monitoring VOCs in atmospheric air I. On-line gas analyzers. Trends in Analytical Chemistry, v.29, n.9, p.1092 - 1100, 2010.

40. KROTKOV, N.A.; CARN, S.A.; KRUEGER, A.J.; BHARTIA, P.K.; YANG, K. Band residual difference algorithm for retrieval of $\mathrm{SO}_{2}$ from the aura ozone monitoring instrument (OMI); IEEE Transactions on Geoscience and Remote Sensing, v.44, n.5, p.1259 - 1266, 2006.

41. KULMALA, M.; VEHKAMAKI, H.; PETAJA, T.; DAL MASO, M.; LAURI, A.; KERMINEN, V.M.; BIRMILI, W.; MCMURRY, P.H. Formation and growth rates of ultrafine atmospheric particles: a review of observations. Journal of Aerosol Science, v.35, p.143 - 176, 2004.

42. LAWRENCE, A.J.; MASIH, A.; TANEJA, A. Indoor/outdoor relationships of carbon monoxide and oxides of nitrogen in domestic homes with roadside, urban and rural locations in a central Indian region. Indoor Air, v.15, n.2, p.76 - 82, 2005.

43. LUVSANA, M-E.; SHIE, R-H.; PUREVDORJ, T.; BADARCH, L.; BALDORJ, B.; CHAN, C-C. The influence of emission sources and meteorological conditions on $\mathrm{SO}_{2}$ pollution in Mongolia. Atmospheric Environment, v.61, p.542 - 549, 2012.

44. MARTINS, E.M.; ARBILLA, G.; BAUERFELDT, G.F.; PAULA, M. Atmospheric levels of aldehydes and BTEX and their relationship with vehicular fleet changes in Rio de Janeiro urban area. Chemosphere, v.67, n.10, p.2096 - 2103, 2007.

45. MURENA, F. Air quality nearby road traffic tunnel portals: BTEX monitoring. Journal of Environmental Sciences, v.19, n.5, p.578 - 583, 2007.

46. PACYNA, J.M. The origin of artic air pollutants: lessons learned and future research. Science of the Total Environment, v.160 - 161, p.39 - 53, 1995.

47. PAN, G.; ZHANG, S.; FENG, Y.; TAKAHASHI, K.; KAGAWA, J.; YU, L.; WANG, P.; LIU, M.; LIU, Q.; HOU, S.; PAN, B.; LI, J. Air pollution and children's respiratory symptoms in six cities of northern China. Respiratory Medicine, v.104, n.12, p.1903 - 1911, 2010.

48. PENGA, Y.P.; CHENA, K.S.; LAIB, C.H.; LUA, P.J.; KAOA, J.H. Concentrations of $\mathrm{H}_{2} \mathrm{O}_{2}$ and $\mathrm{HNO}_{3}$ and $\mathrm{O}_{3}-\mathrm{VOCs}-\mathrm{NO}_{\mathrm{x}}$ sensitivity in ambient air in southern Taiwan. Atmospheric Environment, v.40, n.35, p.6741 - 6751, 2006.

49. POPE III, C.A. Air pollution and health-good news and bad. New England Journal Medicine, v.351, n.11, p.1057 - 1067, 2004.

50. RAIVONEN, M.; VESALA, T.; PIRJOLA, L.; ALTIMIR, N.; KERONEN, P.; KULMALA, M.; HARI, P. Compensation point of $\mathrm{NO}_{x}$ exchange: net result of $\mathrm{NO}_{x}$ consumption and production. Agric. for Meteorol., v.49, n.6, p.1073 - 1081, 2009.

51. SAMET, J.M.; DOMINICI, F.; CURRIERO, F.C., COURSAC, I.; ZEGER, S.L. Fine particulate air pollution and mortality in 20 US cities, 1987 - 1994. New England Journal Medicine, v.343, n.24, p.1742 - 1749, 2000.

52. SARAVANAN, S.; NAGARAJAN, G.; ANAND, S.; SAMPATH, S. Correlation for thermal $\mathrm{NO}_{\mathrm{x}}$ formation in compression ignition $(\mathrm{Cl})$ engine fuelled with diesel and biodiesel. Energy, v.42, n.1, p.401 - 410, 2012.

53. SINGH, H.B.; SALAS, L.; VIEZEE, W.; SITTON, B.; FEREK, R. Measurement of volatile organic chemicals at selected sites in California. Atmospheric Environment, v.26, n.16, p.2929 - 2946, 1992. 
54. SORENSEN, M.; AUTRUP, H.; MOLLER, P. Linking exposure to environmental pollutants with biological effects. Mutat. Res., v.544, n.2 - 3, p.255 - 271, 2003.

55. SUN, Y.; WANG, L.; WANG, Y.; QUAN, L.; ZIRUI, L. In situ measurements of $\mathrm{SO}_{2}, \mathrm{NO}_{\mathrm{x}}, \mathrm{NO}_{y}$, and $\mathrm{O}_{3}$ in Beijing, China during august 2008. Science of the Total Environment, v.409, n.5, p.933 940, 2011.

56. TOSHIAKI, Y. e ICHIRO, M. A case study on identification of airborne organic compounds and time courses of their concentrations in the cabin of new car for private use. Environment International, v.32, n.1, p.58 - 79, 2006.

57. TRAVERSI, D.; DEGAN, R.; DE MARCO, R.; GILLI, G.; PIGNATA, C.; VILLANI, S.; BONO, R. Mutagenic properties of $\mathrm{PM}_{2,5}$ urban pollution in the northern Italy: The nitro-compounds contribution. Environment International, v.35, n.6, p.905 - 910, 2009.

58. US Department of Labor. Occupational Safety and Health Administration. Regulations (Standards - 29 CFR) 1910 Subpart Z - Toxic and Hazardous Substances.

59. VERHEGGEN, B.; MOZURKEWICH, M. Determination of nucleation and growth rates from observation of a $\mathrm{SO}_{2}$ induced atmospheric nucleation event. Journal of Geophysical Research, v.107, n.11, p. AAC 5-1 - AAC 5-12, 2002.

60. VIANA, M.; KUHLBUSCH, T.A.J.; QUEROL, X.; ALASTUEY, A.; HARRISON, R.M.; HOPKE, P.K.; WINIWARTER, W.; VALLIUS, M.; SZIDAT, S.; PRÉVÔT, A.S.H.; HUEGLIN, C.; BLOEMEN, H.; WÅHLIN, P.; VECCHI, R.; MIRANDA, A.I.; KASPER-GIEBL, A.; MAENHAUT, W.; HITZENBERGER, R. Source apportionment of particulate matter in Europe: a review of methods and results. Journal of Aerosol Science, v.39, n.10, p.827 - 849, 2008.

61. VIEIRA, S.E.; STEIN, R.T.; FERRARO, A.A.; PASTRO, L.D.; PEDRO, S.S.C.; LEMOS, M.; SILVA, E. R.; SLY, P. D.; SALDIVA, P. H. Urban air pollutants are significant risk factors for asthma and pneumonia in children: The influence of location on the measurement of pollutants. Arch. Bronconeumol., v. 48, n.11, p. 389 - 395, 2012.

62. WANG, D.K.W. e AUSTIN, C.C. Determination of complex mixtures of volatile organic compounds in ambient air: canister methodology. Analytical and Bioanalytical Chemistry, v.386, n.4, p.1099 - 1120, 2006.

63. WANG, M.; ZHU, T.; ZHANG, J.P.; ZHANG, Q.H.; LIN, W.W.; LI, Y.; WANG, Z.F. Using a mobile laboratory to characterize the distribution and transport of sulfur dioxide in and around Beijing. Atmospheric Chemistry and Physics, v.11, n.6, p.11631 - 11645, 2011.

64. WANG, T.; CHEUNG, T.F.; LI, Y.S.; YU, X.M.; BLAKE, D.R. Emission characteristics of CO, $\mathrm{NO}_{\mathrm{x}}$ $\mathrm{SO}_{2}$ and indications of biomass burning observed at a rural site in eastern China. Journal of Geophysical Research, v.107, n.D12, p. ACH 9-1 - ACH 9-10, 2002.

65. WHO. World Health Organization. Air Quality Guidelines Global Update 2005, E90038. Copenhagen, Denmark, 2006. 496 p.

66. World Health Organization. Europe Particulate Matter Air Pollution: How It Harms Health; 2005. p. 1 - 4.

67. XIAOLIN, L.; YUANXUN, Z.; MINGGUANG, T.; JIANGFENG, L.; LIANGMAN, B.; GUILIN, Z.; YAN, L.; ATSUO, I. Atmospheric lead pollution in fine particulate matter in Shanghai, China. Journal of Environmental Sciences, v.2, n.1118 - 1124, 2009. 
68. YANG, S.; YUESI, W.; CHANGCHUN, Z. Measurement of the vertical profile of atmospheric $\mathrm{SO}_{2}$ during the heating period in Beijing on days of high air pollution. Atmospheric Environment, v.43, n.2, p.468 - 472, 2009.

69. ZHANG, Y.; MU, Y.; LIU, J.; MELLOUKI, A. Levels, sources and health risks of carbonyls and BTEX in the ambient air of Beijing, China. Journal of Environmental Sciences, v.24, n.1, p.124 130, 2012.

70. ZIELINSKA, B.; SAGEBIEL, J.C.; HARSCHFIELD, G.; GERTHER, A.V.; PIERSON, W.R. Volatile organic compounds up to $C_{20}$ emitted from motor vehicles; measurement methods. Atmospheric Environment, v.30, n.12, p.2269 - 2286, 1996. 